\title{
Un ejemplo de producción artesanal en el Mundo Romano: el uso de tegulae con inscipcion $m$ petrvcidivs en Carteia (San Roque, Cádiz)
}

An example of traditional production in the Roman World: the use of tegulae with inscipcion $m$ petrvcidivs in Carteia (San Roque, Cádiz)

Salvador Bravo JimÉneZ ${ }^{1}$

\begin{abstract}
RESUMEN
ABSTRACT

Desde el siglo XVIII se vienen efectuando hallazgos en la zona conocida como el Rocadillo, donde se ubica la antigua ciudad de Carteia, de tegulae con unas curiosas inscripciones donde aparecen el nombre de dos personajes de las que damos información en este trabajo.

PALABRAS CLAVE: From the 18th century they come effecting findings in the zone known as the Rocadillo, where Carteia's former city is located, of tegulae with a few curious inscriptions where they appear the name of two prominent figures of that we give information in this work.

KEYWORDS:

Artesania, Rocadillo, Tegulae, Carteia, Petrucidius.

Artisan, Rocadillo, Tegulae, Carteia, Petrudidus.
\end{abstract}

\section{NTRODUCCIÓN}

Desde que se iniciaron las excavaciones arqueológicas en el Enclave Arqueológico de Carteia (Figuras 1 a 3), el yacimiento ha deparado multitud de hallazgos de todo tipo. Entre estos nos encontramos con una serie de inscripciones sobre tegulae en los que aparece el nombre de la ciudad.

1 Centro Asociado a la UNED en el Campo de Gibraltar. Centro Cívico La Reconquista, s/n. 11202 Algeciras (Cádiz). salva@algeciras.uned.es. 


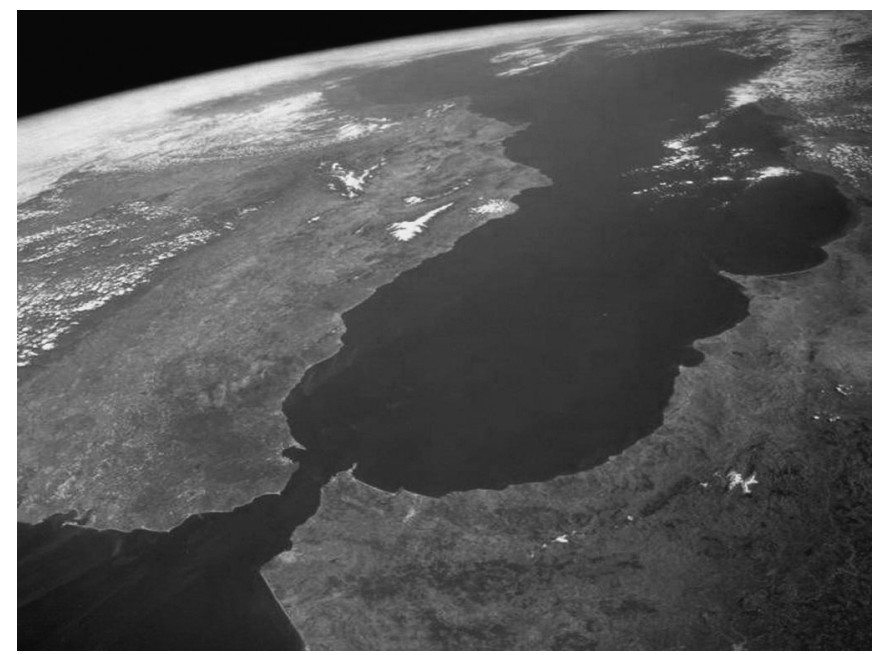

Fig. 1. Carteia en el estrecho de Gibraltar.

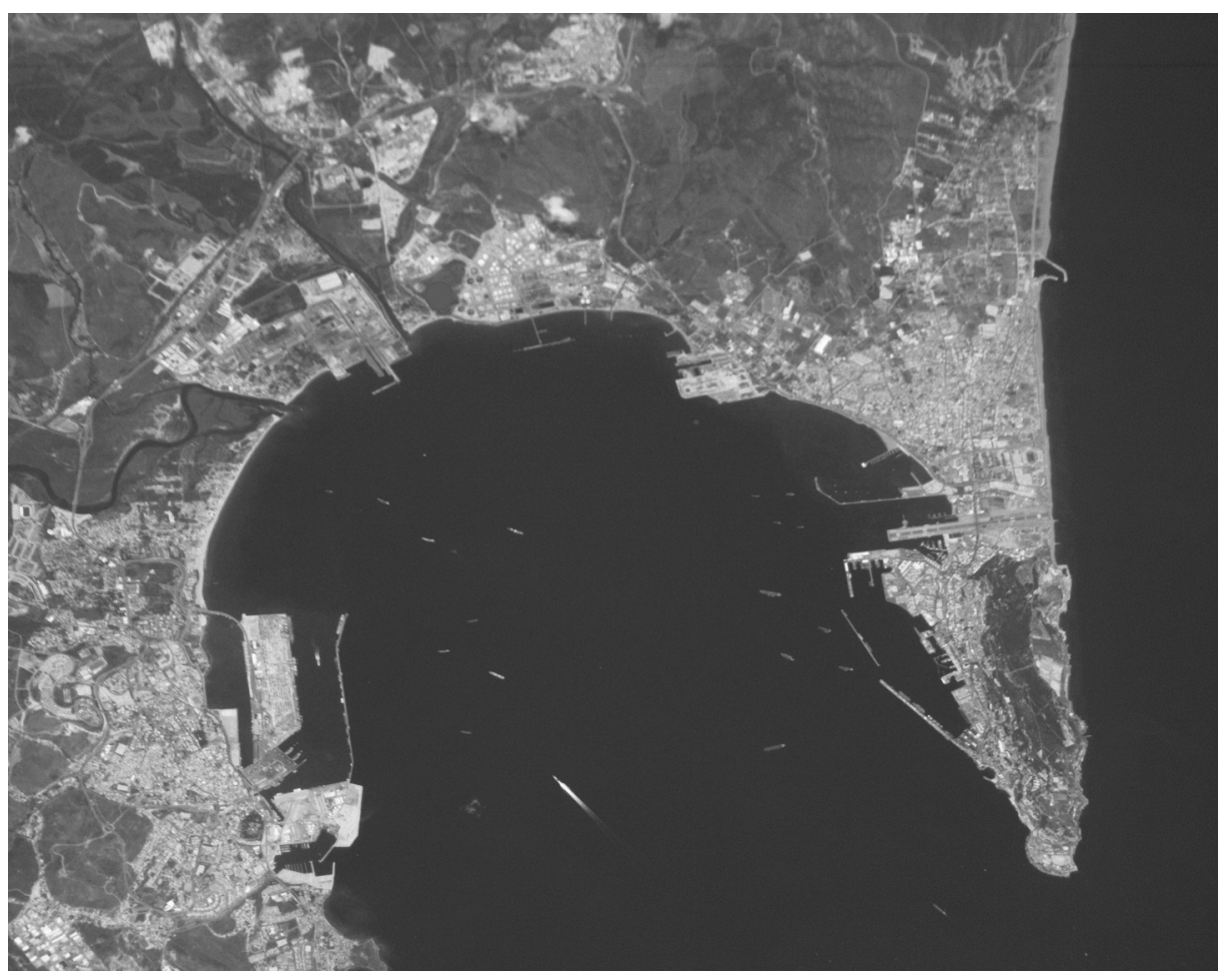

Fig. 2. Carteia en la bahía de Algeciras. 


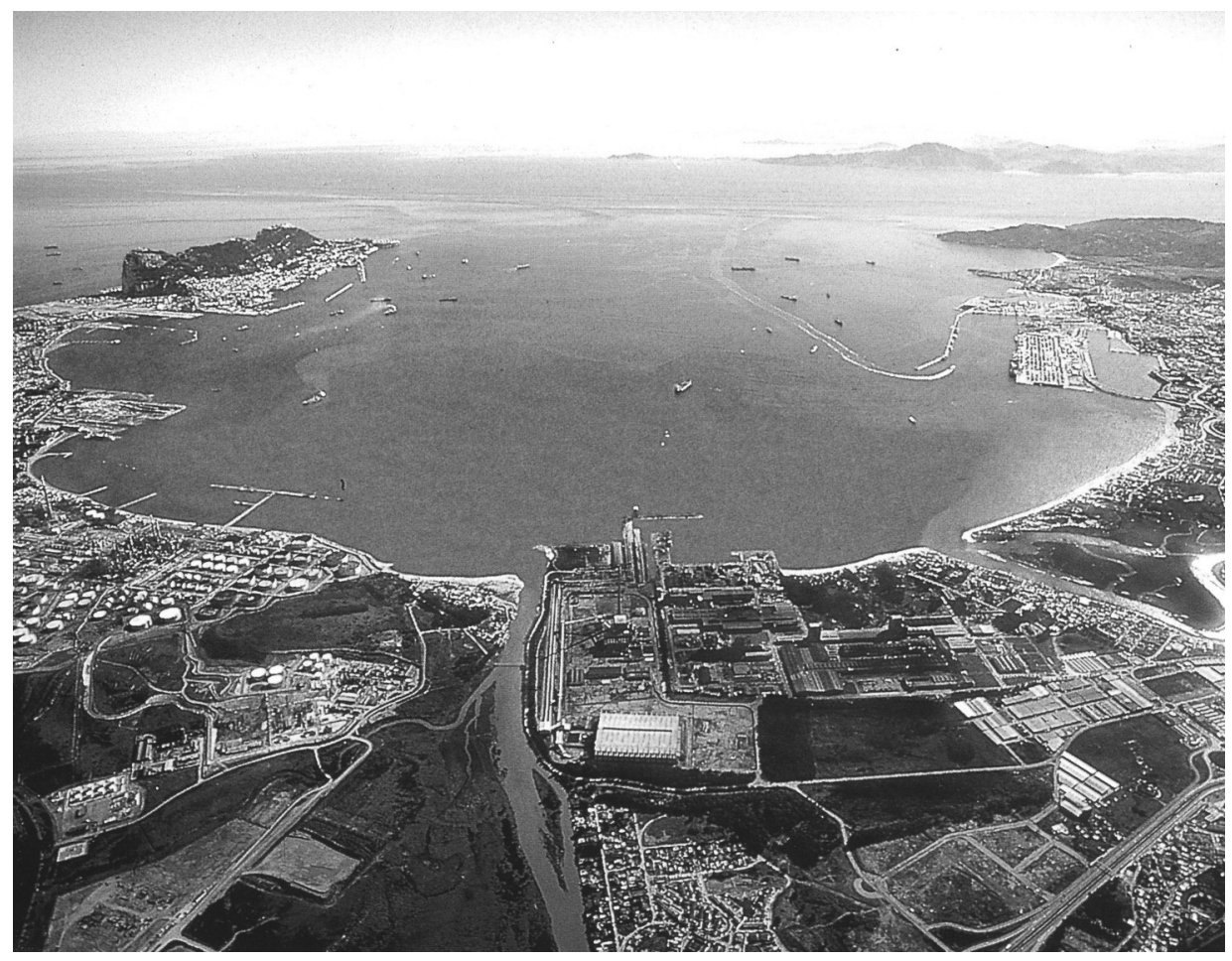

Fig. 3. Emplazamiento de Carteia.

La ciudad de Carteia a lo largo de su devenir histórico se ha caracterizado por dar acomodo a un «cinturón industrial» que la rodea especialmente dedicado a la fabricación de productos cerámicos (ánforas, material de construcción, etc) y salazones ${ }^{2}$. Así, a los conocidos restos salazoneros encuadrados en la zona conocida como Jardín Romántico dentro del propio Enclave Arqueológico (GARCíA DÍAZ, M. y GÓMEZ ARROQUIA, Mํㅡ. I., 2008:121y ss) ya conocidos desde los trabajos acometidos en la zona por Julio Martínez-Santaolalla en la década de 1950, habría que sumar los restos de una figlina hallados en Villa Victoria (BERNAL CASASOLA, D., DÍAZ, J. J., ROLDÁN GÓMEZ, L., BLÁNQUEZ PÉREZ, J. y PRADOS FERNÁNDEZ, F., 2006:245) y la factoría hallada en la zona meridional de la ciudad en 2008 (GARCÍA PANTOJA, Maㅡ E, EXPÓSITO ÁLVAREZ, J. A. y MONCAYO MONTERO F. J., 2010:257 y ss.)

Por lo que se refiere a los alfares, los avances llevados a cabo en este aspecto son notables (TOMASSETTI GUERRA, J. Mª y BRAVO JIMÉNEZ, S., 2006:254

2 Tema ampliamente tratado en BRAVO JIMÉNEZ, S. (2010): Dinámicas de control ideológico y territorial en el estrecho de Gibraltar en épocas fenicia, púnica y romana. http://espacio.uned.es/fez/view.php?pid=tesisuned:GeoHis-Sbravo. 
y ss). Así, en Arroyo de las Cañas, se encuentra el complejo alfarero de Venta del Carmen (Los Barrios), descubierto por De Vicente y Pecino a principios de los años 80 (en BERNAL CASASOLA, D. 1998:28). Este complejo ya era conocido desde mucho antes; así, Ponsich cita el hallazgo de un horno y producciones anfóricas encuadradas en la 1a mitad del siglo I (PONSICH, M., 1988:66 y ss). Se han llevado a cabo dos intervenciones arqueológicas poniendo de manifiesto la importancia del lugar merced al hallazgo de dos hornos de planta circular con varias estructuras relacionadas enmarcados en el tránsito entre el siglo I a. C. y el I.

A unos 2 kilómetros de Venta del Carmen se produjo el hallazgo, en la década de los 80 en los terrenos pertenecientes a la factoría CAMPSA, de un horno que se encontraba, al parecer, parcialmente destruido (BERNAL CASASOLA, D., 1997:68) de planta posiblemente circular ${ }^{3}$. En el entorno de ambos, en la década de los setenta, el prof. Beltrán documentó un horno alfarero con clara vinculación a Carteia y que el citado investigador dató a finales del siglo I. Se encontraba muy cerca de la línea de costa (BELTRÁN, M, 1977;223).

Más al Este, nos encontramos con el alfar romano de la Calle Aurora (FERNÁNDEZ CACHO, S, 1994; 29). Se evidenció merced al hallazgo de un testar. Para Silvia Fernández, fue uno de los talleres emisores de ánforas de la zona (FERNÁNDEZ CACHO, S, 1997:179; LAGÓSTENA BARRIOS, L. y BERNAL CASASOLA, D., 2004:81).

Más alejado se encuentra el Cortijo de Albalate, a unos $2 \mathrm{Km}$ del núcleo urbano de San Roque. Según la noticia de Daniel Sedeño (en BERNAL CASASOLA, D., 1998; 33) en su lugar hubo un horno para la fabricación de cerámicas que se cubrió. En 1997, según Bernal, una oquedad en el suelo permitió documentar los restos de un horno de planta rectangular en muy buen estado de conservación con toda la cámara de combustión completa y la parrilla sustentada por falsos arcos de ladrillo. Dada la gran amplitud de los hallazgos en la zona, quizá estemos ante un complejo de cierta importancia datado en época altoimperial.

Por último, en la zona de Puente Mayorga conocida como Villa Victoria, salieron a relucir restos de un alfar datado en la la mitad de siglo I (BLÁNQUEZ PÉREZ, J. et alii, 2004:461)

Vista la dispersión de las figlinae en la zona, caben algunas consideraciones.

En primer lugar, la riqueza geomorfológica de la zona hace viable la instalación de hornos a lo largo de la costa donde a la magnífica calidad de las arcillas hay que sumar la abundancia de agua materializada en los numerosos cursos fluviales que desembocan en la zona. Estamos pues ante un paisaje con una enorme potencialidad para el desarrollo de industrias alfareras, como puede apreciarse en la figura 4.

3 Este horno permanece en relativo buen estado de conservación aunque expuesto a los riesgos derivados de los agentes climáticos que lo han erosionado en parte. Se sitúa muy próximo al horno de Las Cañadas. 


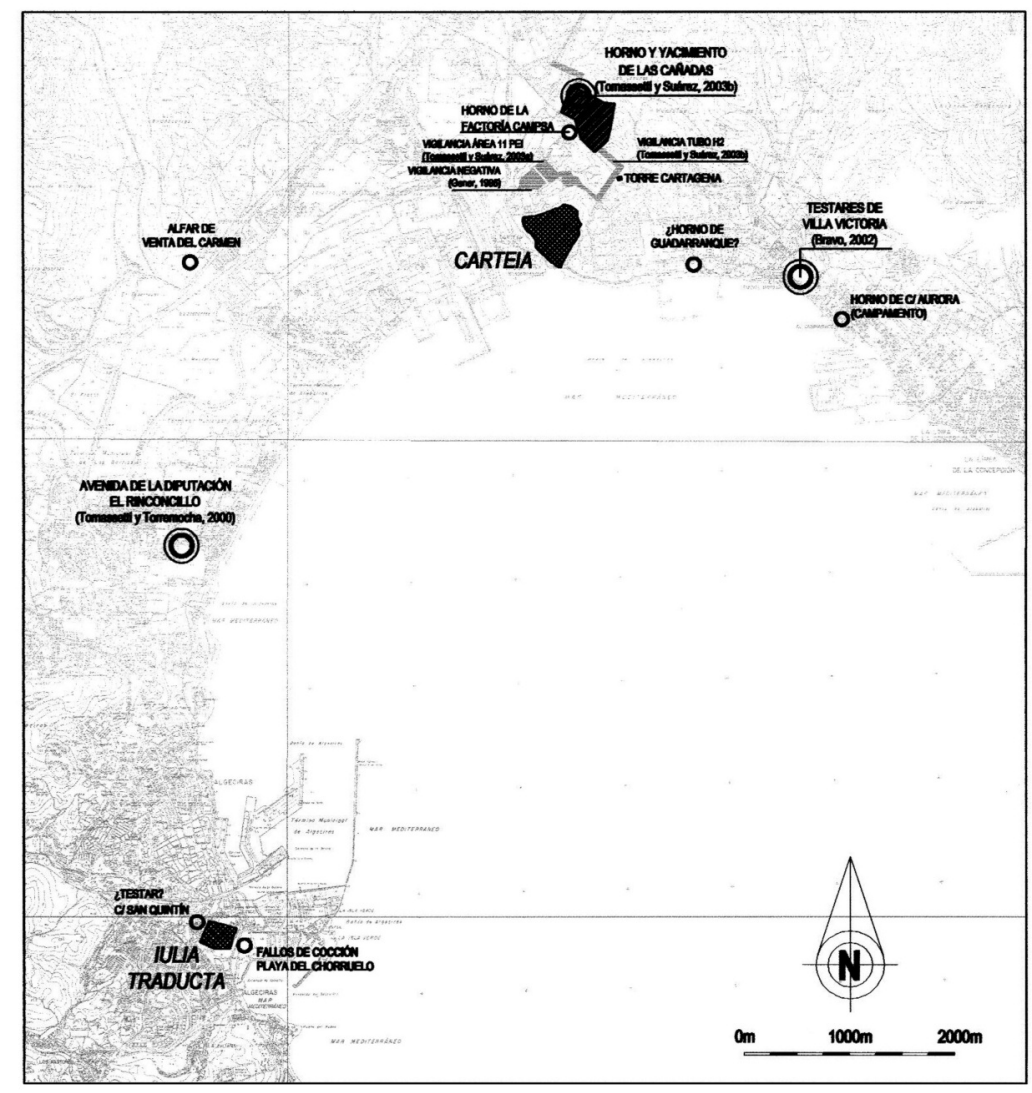

Fig. 4. Alfares romanos en la bahía de Algeciras (TOMASSETTI GUERRA, J. Maㅡ y BRAVO JIMÉNEZ, S., 2006:253).

Por otro lado, la existencia de Carteia, verdadero foco de romanidad en el Campo de Gibraltar desde la primera mitad del siglo II a. C. (BRAVO JIMÉNEZ, S. en prensa) hace que se den las condiciones idóneas para el establecimiento industrial. El hecho de encontrarse en una encrucijada de caminos como lo es el estrecho de Gibraltar hará de Carteia uno de los focos irradiadores no solo de cultura romana hacia el interior del país, sino de centro distribuidor de productos derivados de la pesca como el garum y sus contenedores. Merece a este respecto traer a colación la cita de Plinio sobre las excelencias de las factorías de salazones de la colonia libertinorum (BRAVO JIMÉNEZ, S., 2002:67). Así, más que para consumo propio, las figlinae analizadas deberían de ponerse en relación con el tráfico comercial por el mediterráneo y el Norte de África. A este respecto es interesante el análisis que hace Enrique Gozalbes sobre la ruta comercial entre Carteia y las ciudades norteafricanas de Septem Fratres y Tamuda (GOZALBES CRAVIOTO, E., 1997:169). 
A finales del siglo I a. C., tras los avatares de la guerra civil entre Octavio y Marco Antonio (31 a. C.) la zona vuelve a sufrir una remodelación territorial merced a la instalación de contingentes poblacionales del Norte de África (GOZALBES CRAVIOTO, E, 1993:70) y a la creación de un nuevo núcleo poblacional con veteranos romanos (BRAVO JIMÉNEZ, S., 2003:114). En efecto, en torno a los años 29 ó 28 a. C. tiene lugar la fundación de lulia Traducta muy probablemente en el núcleo Sur de la ciudad de Algeciras (BRAVO JIMÉNEZ, S., 2003: 115; BERNAL CASASOLA, D. y JIMÉNEZ-CAMINO ÁLVAREZ, R., 2007:161). Tras la fundación del nuevo núcleo y el declive lógico de Carteia 4 por apoyar al bando perdedor, el territorium de Carteia se verá remodelado en su zona occidental de la mano de la nueva ciudad por lo que es muy probable que dejara de gestionar los territorios inmediatos a la zona donde se ubica actualmente la ciudad de Algeciras. Así, los hornos de la playa del Chorruelo y los de la Calle San Quintín, se enmarcarían en el ámbito de Traducta. Queda por dilucidar el complejo alfarero de El Rinconcillo, aunque muy probablemente tras la guerra civil y el consiguiente reparto de territorio, quedaría vinculado a la ciudad de Traducta al igual que los recientemente excavados en la Factoría Garavillas (TOMASSETTI GUERRA. J., Maㅡ, TORREZ ABRIL, F. L., SUÁREZ PADILLA, J., MARTÍN ESCARCENA, A. Mํㅡㄹ AYALA LOZANO, S. y ÁLVAREZ GONZÁLEZ, R., 2009:101; ÁLVAREZ GONZÁLEZ, R., TOMASSETTI GUERRA, J. $M^{a}{ }^{a}$, MARTÍN ESCARCENA, A. M ${ }^{a}$, AYALA LOZANO, S. y SUÁREZ PADILLA, J., 2008:76).

Lo cierto es que nos encontramos ante un gran foco productor alfarero de primera magnitud cuyas producciones parecen no centrarse únicamente en el mercado local, sino que aprovechan su inmejorable situación estratégica para aprovecharlo comercialmente. Las producciones deben de entenderse bajo esa óptica exportadora en un momento (mediados del siglo I) de clara expansión del mercado del garum e industrias derivadas. No obstante, otras producciones como el vino o el aceite no deben ser descartadas. Los envases fabricados en los alfares así lo prueban.

\section{LOS PERSONAJES}

En las tegulae que nos interesan, aparecen escritos los nombres de dos personajes de los que intentaremos conocer quienes eran y a qué se dedicaban. El primero de ellos es Marcus Petrucidius. Este personaje ha estado sujeto a fuerte controversia ya desde la aparición de las primeras inscripciones sobre tegulae que se llevaron a cabo en torno a 1860. Para Hübner, el personaje fue legado de Marco Licinio, procónsul de la Bética (CIL II 4967, 1), pero este argumento fue refutado por Dessau (DESSAU, H., 1914:58) al asociar otro procónsul a Petrucidius sosteniendo que sería o bien un tegularius o bien un conductor operarum, legado

4 Carteia tomó partido por el bando senatorial en la guerra civil apoyando a los hijos de Pompeyo en su lucha contra Octavio. Bellum Hispaniense, 32: "Cn. Pompeius cum equitibus paucis nonnullisque peditibus ad navale praesidium Carteiam contendit, quod oppidum abest ab Corduba milia passum CLXX». 
Un ejemplo de producción artesanal en el Mundo Romano: el uso de tegulae...

de Gneo Pompeyo aunque este hecho solamente se sostiene por la especial vinculación de Carteia a Pompeyo.

Sobre Marco Licinio todo apunta a que sería un tegularius o un conductor operarum que trabajaría a las órdenes de Marco Petrucidio pues su nombre solamente aparece en la zona gaditana, en concreto en Hasta Regia y Carteia. Por último, en opinión de Julián González (GONZÁLEZ, J. 1989:523) Petrucidio debió de ser un legatus pro praetore y Marco Licinio su conductor o tegularius en torno a los años 15 ó 14 a. C. o inmediatamente anteriores debido a varias circunstancias que trataré de analizar a continuación.

Pero para ello debemos de analizar los hallazgos donde han aparecido dichos epígrafes sobre tegulae.

\section{LAS TEGULAE}

En 1982, en la memoria de las excavaciones arqueológicas llevadas a cabo en el Enclave Arqueológico de Carteia bajo la dirección del Prof. Presedo Velo, se hace un somero análisis de estas piezas que han ido apareciendo en los trabajos arqueológicos llevados a cabo durante la década de 1970.

El citado catedrático de la Universidad de Sevilla llevó a cabo un profundo estudio en la citada memoria, a la cual me remito

Son piezas muy características que responden al tipo denominado tegulae bipedales (de FILIPPO, R., 2004:97) de doble pestaña de 60 X $45 \mathrm{~cm}$ aproximadamente y 2, 5 centímetros de grosor. Las pestañas suelen tener entre 4, 5 y 5 centímetros de altura.

Los primeros hallazgos de los que tenemos constancia se produjeron en la propia Carteia en 1860 y 1865 donde las tegulae que contenían la inscripción:

M. PETRVCIDIVS. M. F. / LEG. PRO PR. M. LICI (CIL III 4967, 1)

fueron llevadas a Gibraltar por un barbero y publicadas por Hübner en el CIL, 1004.

Más tarde, el propio erudito alemán recogía nuevos hallazgos en Alcalá del Río (Sevilla) con la particularidad de la ausencia de Marco Licinio, hecho que me parece de enorme importancia como luego veremos a los que se sumó un nuevo hallazgo de similares características en Itálica. En dichas tegulae, la inscripción era:

[M(ARCUS) PE]TRUCIDIUS) / PRO PR(AETORE) (CILA II, 332)

y la italicense:

M(ARCUS) PETR]UCIDIUS M(ARCI) F(ILIUS) / [LEG(ATUS) PR]O

PR(AETORE) ALEX(ANDER?) ST(RUXIT?) (CIL 01 2298B p 1113)

(C) UNED. Espacio, Tiempo y Forma

Serie II, Historia Antigua, t. 24, 2011 


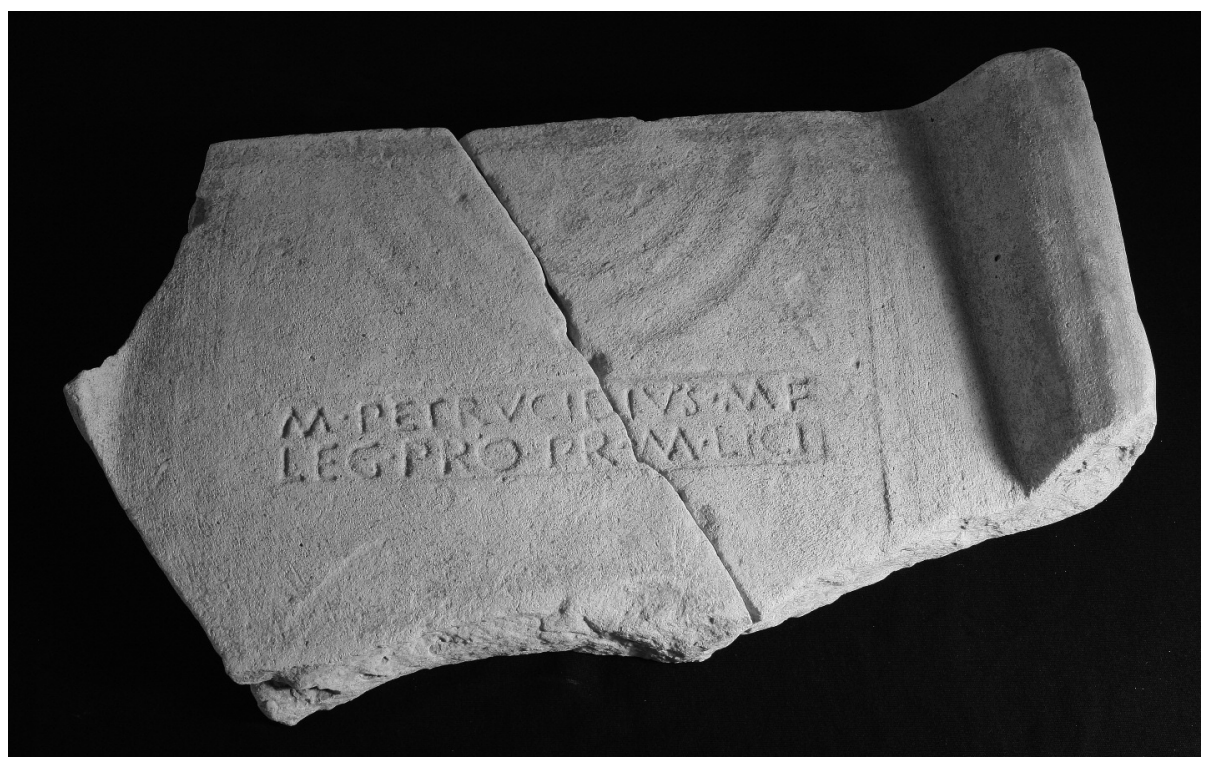

Fig. 5. Tegula con inscripción M Petrucidius, hallada en las excavaciones efectuadas entre 1971 y 1973.

A la que habría de sumar una nueva aparecida en 1985 en Utrera (Sevilla): M(ARCUS) PETRUCIDIUS M(ARCI) F(ILIUS) / LEG(ATUS) PRO PR(AETORE) // CN(AEI) TARQ(UINII?) (CILA II, 955)

Por ultimo, en 1909 ya habían aparecido en Mesas de Asta (Jerez de la Frontera, Cádiz) dos inscripciones de similares características a las de Carteia:

M(ARCUS) PETRU[CIDIUS M(ARCI) F(ILIUS) / LEG(ATUS) PRO P[R(AETORE) M(ARCI) LICINI] // [M(ARCUS) PETR]UCIDIUS M(ARCI) F(ILIUS) / [LEG(ATUS) PR]O PR(AETORE) M(ARCI) LICI[NI] (IRPCADIZ 33) (GONZÁLEZ, J., 1982:27)

Vistos estos antecedentes, las posturas de los investigadores que han estudiado el tema no han llegado a una conclusión unitaria sino que, a mi modo de ver, han arrojado más confusión al problema.

Para Hübner, Marco Petrucidio sería el legado de un Procónsul de la Bética de nombre Marco Licinio (HÜBNER, E., 1869:395); sin embargo, el hecho de que aparecieran las tegulae de la zona sevillana con los nombres de Alexander y Tarquinio asociados a M. Petrucidio llevó a Dessau a proponerlo como legado del propio Pompeyo (DESSAU, H., 1913:131) siendo Alexander el encargado por Petrucidio de construir las tegulae. La adscripción pompeyana de las tegulae realizada por Dessau se fundamentaba únicamente en la estrecha relación entre Carteia y Pompeyo rechazando de plano que un procónsul de la Bética de nombre Marco Li- 
Un ejemplo de producción artesanal en el Mundo Romano: el uso de tegulae...

cinio. De similar parecer es Marchetti aunque elevando a Petrucidio al rango de gobernador de la Provincia Ulterior mientras que Licinio sería un conductor operarum (MARCHETTI, M., 1922:905).

Varios autores han seguido dando explicaciones al respecto como Alföldy, Münzer, Petersen, Broughton, Szramkiewicz o Miltner aunque la mayoría, como Presedo (PRESEDO VELO, F. J., MUÑIZ COELLO, J., SANTERO SANTURINO, J. Ma y CHAVES TRISTÁN, F., 1982:279) han seguido de una u otra forma las tesis de Dessau ${ }^{5}$.

El análisis que hace Presedo tras sus campañas de excavación en el yacimiento es bastante concluyente a mi parecer toda vez que las tegulae aparecen en contextos estratigráficos. El catedrático de la Universidad hispalense encontró dos tegulae en la zona de la Torre del Rocadillo y en el Cortijo en pleno foro de la ciudad. Fechó ambas a finales del periodo republicano, tras la guerra civil que es cuando sugiere que se lleva a cabo una amplia remodelación urbanística de la ciudad (PRESEDO VELO, F. J., MUÑIZ COELLO, J., SANTERO SANTURINO, J. Mª y CHAVES TRISTÁN, F., 1982:281). Para Presedo, siguiendo a Münzer (RE, XIX, 2, col. 1304-5), Marco Petrucidio sería oriundo del Piceno y partidario de Pompeyo en la guerra civil.

Para Julián González (GONZÁLEZ, J. 1989:523), la actividad constructora que motiva la adquisición de las tegulae no debe enmarcarse al final de la guerra entre Pompeyo y César, sino al final de la guerra civil entre Antonio y Octavio y más concretamente en Actium en 31 a. C. Me uno plenamente a este razonamiento apoyándome en la intensa actividad constructora que soporta la ciudad durante el periodo augusteo con la construcción de un nuevo templo, un complejo termal y un teatro además de la llamada domus del Rocadillo recientemente excavada6 (BRAVO JIMÉNEZ, S., 2010).

La fundación de lulia Traducta en 29 ó 28 a. C. frente a Carteia (BRAVO JIMÉNEZ, S., 2003:118) conllevará la necesidad de remodelar urbanísticamente la antigua ciudad de Carteia con el consiguiente empleo de tegulae de uso doméstico, tegulae que solamente aparecen en ciudades que de una u otra forma han adquirido un rango sea de municipio o colonia. Quizás, siguiendo la hipótesis del prof. González, Marco Petrucidio sería nada más que un funcionario encargado de esa remodelación urbanística preconizada por Octavio, ya Augusto a partir del último decenio del siglo I a. C. (GONZÁLEZ, J. 1989:523).

5 Para un mayor conocimiento sobre el parecer de estos autores ver el trabajo de GONZALEZ, J. (1989): «M. Petrucidius M. f. legatus pro pr.» Athenaeum, 67. Pavia. pp. 517- 524.

6 Durante el mes de noviembre de 2010 tuve la ocasión de llevar a cabo una Actividad Arqueológica Puntual en la llamada Domus del Rocadillo concluyendo que el edificio se encontraba ya en pleno uso en la primera mitad del siglo I para pasar por una serie de avatares y reformas constructivas hasta mediados del siglo II cuando el decumanus se encuentra ya amortizado por basuras. 


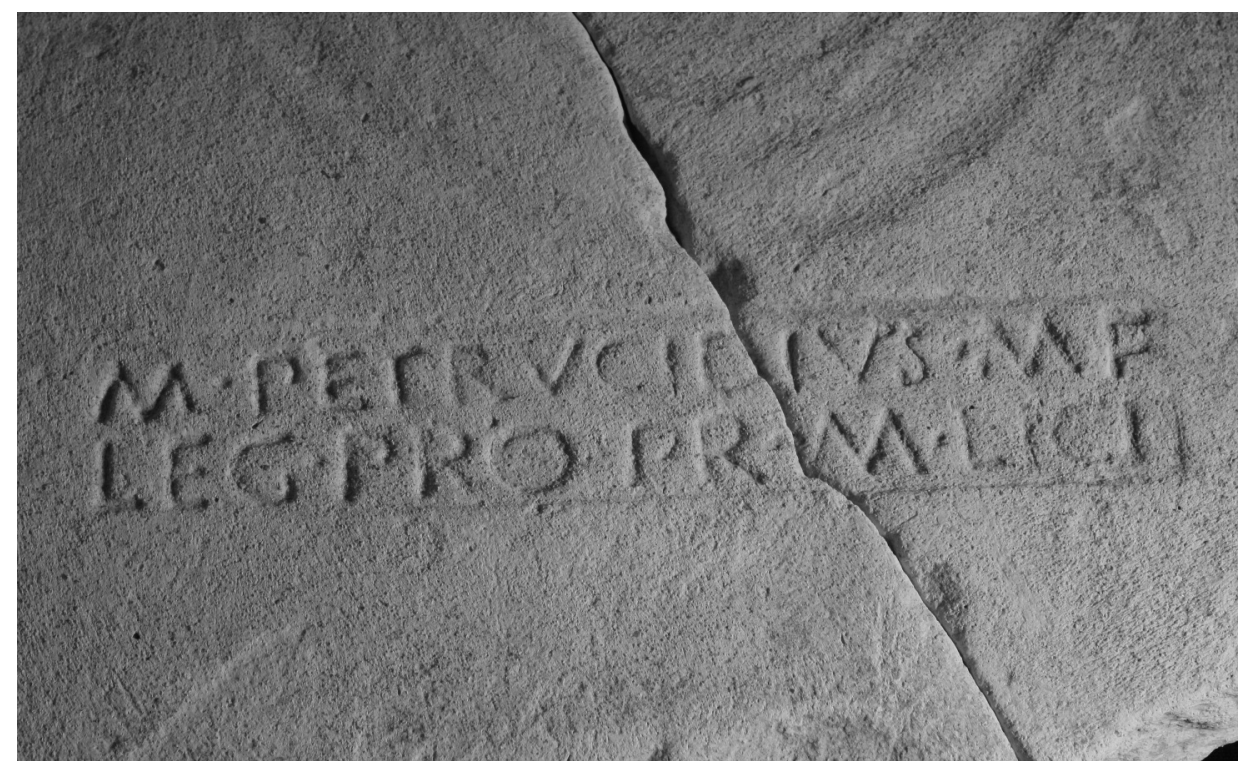

Fig. 6. Inscripción M Petrvcidivs M f Leg. Pro Pr M Lici.

\section{CONCLUSIONES}

Es sintomático que estamos ante una zona donde la profusión de asentamientos dedicados a la misma actividad hace que debamos plantearnos sobre la funcionalidad de los mismos. El hecho que aparezcan tantos hornos de la misma época con una ratio $\mathrm{Km}^{2}$ tan pequeña no es casual y responde a una disposición del territorio perfectamente establecida desde los dos grandes núcleos poblacionales en época altoimperial: Iulia Traducta y Carteia.

Si exceptuamos la planta del horno del Cortijo de Albalate, la adscripción del horno de El Rinconcillo al tipo Ilb de Cuomo di Caprio se constituye como el primer ejemplo de este tipo de hornos en el Campo de Gibraltar. Es interesante destacar la tipología de los hornos rectangulares pues, como apuntan Bernal y Jiménez-Camino (BERNAL CASASOLA, D. y JIMÉNEZ-CAMINO ÁLVAREZ, R., 2004:592), el hecho de encontrarse un horno con esta estructura estaría íntimamente relacionado con población itálica en la zona. Creo no obstante que es arriesgado vincular tipología de los hornos con población toda vez que parece que no se cumplen estos parámetros en otras zonas de la Península Ibérica ${ }^{7}$ y sí vincular tipología de los hornos con especialización productiva.

7 Son los hornos de planta rectangular los que priman en el territorio de la Tarraconense así como en Bretaña y el sur de Galia. Aunque el aporte poblacional itálico fue importante en estas regiones, no parece que podamos vincular dichos hornos con emigrantes itálicos. 
Un ejemplo de producción artesanal en el Mundo Romano: el uso de tegulae...

Dicho tipo de horno es de planta rectangular con un corredor central ampliamente utilizado en todo el territorio romano, desde Italia hasta Britania. A destacar la poca presencia en la Bética si exceptuamos el cercano del Cortijo de Albalate y un horno de pequeñas dimensiones excavado en el Saladillo (Estepona, Málaga) dedicado a la fabricación de Tegulae (SUÁREZ PADILLA, J., TOMASSETTI GUERRA, J. Maㅡ, BRAVO JIMÉNEZ, S., FERNÁNDEZ RODRIGUEZ, L. E. y NAVARRO LUENGO, I., 2004: 717). También se detectó un horno de similar tipología en Bobadilla (Málaga) y otro en Calle Carretería en la ciudad de Málaga que conviviría con uno de planta circular (RAMBLA TORRALBO, J. A. y MAYORGA MAYORGA, J., 1997:75), aunque ambos fueron destruidos.

Lo cierto es que parece que su dedicación es polivalente aunque prima la fabricación de materiales de construcción (como demuestra el horno de Estepona) y de cerámica común. En Galia (LE NY, 1988:69 y ss), Italia (CUOMO DI CAPRIO, N., 1971-1972: 444 y ss) y Britania (MCWHIRR, A., 1979:97 y ss) es con diferencia, el tipo mayoritario y por lo que respecta a Hispania, es en la Tarraconense y concretamente en la región de Gerona donde su porcentaje se acerca al $80 \%$ de los hornos conocidos (TREMOLEDA, J.,1995:88) aunque lo vemos en zonas del interior como el horno de La Jericó en Herrera de Pisuerga (Palencia) (PÉREZ, C., 1989:246 y ss).

También tenemos documentados hornos de planta rectangular en el Viaducto del Pretorio en Córdoba (MORENO, M. y VARGAS, S., 2004; e. p.) con la salvedad que sus excavadores indican que predominaba el tipo de material de construcción entre sus producciones.

De confirmarse el binomio horno de planta cuadrada -producción de materiales de construcción-, estaríamos ante el único ejemplo de producción conocida de material constructivo en la bahía de Algeciras. Este hecho parece confirmarlo el horno romano de Villamanta (Madrid) la antigua Mantua, donde Mar Zarzalejos (ZARZALEJOS PRIETO, M., 2002:75 y ss) identificó y excavó dos hornos del tipo Ilb de Cuomo di Caprio similares al de la Avda. de la Diputación. Para eludir en la medida de lo posible las pérdidas de calor, se procedió a excavar la cámara de combustión en el subsuelo, recurso que, como hemos visto, se llevó a cabo en el horno del Rinconcillo y que señala Cuomo di Caprio como recurrente ante las posibles pérdidas caloríficas (CUOMO DI CAPRIO, N., 1971-1972:389). La dedicación del horno a la producción de ímbrices así lo corrobora.

El hecho de repetirse en los hornos rectangulares la fabricación de materiales constructivos nos parece de suma importancia en la caracterización que estamos haciendo de los hornos en el arco Norte de la bahía algecireña y vinculadas a Carteia. El descubrimiento del vertido de la figlina en Villa Victoria nos permite decantarnos por la producción anfórica y de cerámicas de cocina para dicha estructura; sin embargo, el hecho de no haber encontrado materiales claramente adscribibles al horno del Rinconcillo hace que nos planteemos su verdadera dedicación.

El hecho de que aparezca en las tegulae los nombres de Marco Petrucidio y Marco Licinio hace que debamos plantearnos una acción constructiva de gran 
envergadura en la zona y de la que Carteia no es ajena: nos referimos a la gran remodelación urbanística que se lleva a cabo en época augustea en el Campo de Gibraltar y de la que las tegulae de Petrucidio no son sino fiel reflejo.

\section{BIBLIOGRAFÍA}

ADAM, J. P. (1996): La construcción romana. Materiales y técnicas. León.

ALBERTOS, Ma L. (1966): Onomástica primitiva de Hispania Tarraconense y Bética. Salamanca.

ÁLVAREZ GONZÁLEZ, R., TOMASSETTI GUERRA, J. Mª̣, MARTÍN ESCARCENA, A. Mª AYALA LOZANO, S. y SUÁREZ PADILLA, J. (2008): Memoria Definitiva de la Actividad Arqueológica Preventiva en Paseo Victoria Eugenia, 13. Antigua fábrica de conservas Garavilla. Algeciras (Cádiz). Delegación provincial de Cultura en Cádiz. Inédito.

BAENA DEL ALCÁZAR, L. (1997): «Arquitectura y tipología de los hornos romanos malacitanos». Figlinae Malacitanae, Málaga.

BELTRÁN LLORIS, M. (1977): «Problemas de la morfología y del concepto histórico-geográfico que recubre la noción tipo. Aportaciones a la tipología de las ánforas béticas». Méthodes classiques et méthodes formelles dans l'étude des amphores (Actes du Colloque de Rome, 27-19 mai, 1974). Collection de L'École Française de Rome, 32: 97-131.

- (1990): Guía de la cerámica romana. Zaragoza.

BEJARANO, M. y PEÑALOSA, B. (inédito): «Informe-Memoria de la intervención arqueológica de urgencia realizada en la parcela $C$ de la urbanización Costalita I. Estepona (Málaga)». Informe administrativo, inédito.

BENDALA GALÁN, M., RICO, C. y ROLDÁN GÓMEZ, L. (1999): El ladrillo y sus derivados en la época romana. Monografías de Arquitectura Romana, 4. Madrid.

BERNAL CASASOLA, D. (1997): «La producción anfórica en la Bahía de Algeciras en época romana: nuevos datos procedentes de los talleres de Venta del Carmen (Los Barrios)». Almoraima, 17. Algeciras. pp. 65-74.

- (1998a): «La producción anfórica en la Bahía de Algeciras en época romana». En BERNAL (ed.): Excavaciones arqueológicas en el alfar romano de la Venta del Carmen. Los Barrios (Cádiz). Madrid, pp. 19-42.

- (1998b): «Las ánforas de producción local: tipología, caracterización y epigrafía». En BERNAL (ed.): Excavaciones arqueológicas en el alfar romano de la Venta del Carmen. Los Barrios (Cádiz). Madrid, pp. 143-198.

BERNAL CASASOLA, D. y JIMÉNEZ-CAMINO ÁLVAREZ, R. (2004): «El taller alfarero de "El Rinconcillo" y la importancia del factor itálico en la bahía de Algeciras (s. I a.c.)». En Figlinae Baeticae. Talleres alfareros y producciones cerámicas en la Bética romana (ss. II a.C-VII d. C). Facultad de Filosofía y Letras. Universidad de Cádiz (Cádiz, 12-14 noviembre 2003). Cádiz. pp. 589-606.

BERNAL CASASOLA, D., DÍAZ RODRIGUEZ, J. J., ROLDÁN GÓMEZ, L., BLÁNQUEZ PÉREZ, J. y PRADOS MARTÍNEZ, F. (2006): «Villa Victoria, una figlina altoimperial en el territorium de Carteia». I Jornadas de Arqueología del Campo de Gibraltar. Protección del Patrimonio. Tarifa, 23-25 de abril de 2004. Instituto de Estudios Campogibraltareños. pp. 235-250.

De FILIPPO, R. (2004): «La brique et les matériaux de construction en terre cuite à l'époque romaine" en BESSAC, J. C. et alii, La construction. Les matériaux durs: pierre et terre cuite. Collection Archéologiques. Paris.

BLÁNQUEZ PÉREZ, J., PRADOS MARTÍNEZ, F., ROLDÁN GÓMEZ, L., DÍAZ RODRÍGUEZ, J. J. y BERNAL CASASOLA, D. (2004): «Villa Victoria y el barrio alfarero de carteia en el s. I d. C.: avance de la excavación del 2003» en LAGÓSTENA BARRIOS, L. y BERNAL CASASOLA, D. (eds): Figlina Baeticae: talleres alfareros y producciones cerá- 
Un ejemplo de producción artesanal en el Mundo Romano: el uso de tegulae...

micas en la Bética romana (ss. II a.C - VII d. C): Actas del Congreso Internacional. Cádiz. 12-14 noviembre de 2003. Vol. II. Cádiz. pp. 457-472.

BRAVO JIMÉNEZ, S. y GUZMÁN, J. C. (2002): Un suceso extraordinario ocurrido en el Campo de Gibraltar a mediados del Siglo II a. n. e.». Eúphoros, Algeciras. pp.

BRAVO JIMÉNEZ, S. (2003a): Informe preliminar de la Intervención Arqueológica de Urgencia en Plan Parcial Villa Victoria, Puente Mayorga (San Roque). Delegación Provincial de Cultura. Cádiz. Inédito.

- (2003b): «Iulia Traducta: ¿Una colonia romana en la bahía de Algeciras?» Actas del Iller Congreso de Historia de Andalucía. Córdoba, 2001. Córdoba. pp. 97-120.

- (2005): «lulia Traducta y Tingi. Dos ciudades romanas en los confines del Imperio». XVo Convegno Internazionale di Studi L'Africa Romana. Tozeur, 11-15 de diciembre de 2002. Universidad de Sassari (Italia). Cagliari. pp. 651-672.

- (2010): Memoria de los trabajos realizados en relación con la Actividad Arqueológica Puntual titulada: limpieza superficial de estructuras en la Domus del Rocadillo en Enclave Arqueológico de Carteia (San Roque, Cádiz). Delegación Provincial de Cultura. Cádiz. Inédito.

- (en prensa): «La deductio de Carteia: un hecho singular ocurrido en el Campo de Gibraltar en el siglo II a. n. e.» XI Jornadas de Historia del Campo de Gibraltar. Jimena de la Frontera, 22 a 24 de octubre de 2010. IECG.

CUOMO DI CAPRIO, N. (1971-1972): «Proposta di classificazione delle fornaci per ceramica e laterizi nell'area italiana dalla prehistoria a tutta l'epoca romana». Riv. Sibrium, XI. pp. $371-464$.

DESSAU, H. (1892-1916): Inscripciones Latinae Selectae. Berlín.

FERNÁNDEZ CACHO, S. (1991): Excavaciones arqueológicas en El Rinconcillo (Algeciras, Cádiz) (Octubre - Noviembre, 1991). Delegación Provincial de Cultura. Cádiz. Inédito.

- (1995): «Excavaciones arqueológicas en El Rinconcillo (Algeciras, Cádiz)». Anuario Arqueológico de Andalucía, III. Año 1992. Sevilla. pp. 70-77.

- (1997a): «Las industrias derivadas de la pesca en la provincia romana de la Bética: la alfarería de El Rinconcillo (Algeciras, Cádiz)». Spal, 4. pp. 173 -214. Sevilla.

- (1997b): Informe arqueológico de la ejecución de obras en el complejo "Residencial Monteverde» (Algeciras, Cádiz). Delegación Provincial de Cultura. Cádiz. Inédito.

FLECHTER, D. (1965): «Tipología de los hornos romanos de España», AEspA, 38. Madrid.

GARCÍA DÍAZ, M. y GÓMEZ ARROQUIA, Mํㅣ. (2008): «La factoría de salazones del Jardín Romántico de Carteia». IX Jornadas de Historia del Campo de Gibraltar. Algeciras. pp. 119-127.

GARCÍA PANTOJA, Mํㅡㄹ EXXÓSITO ÁLVAREZ, J. A. y MONCAYO MONTERO F. J. (2010): «El barrio industrial salazonero de Carteia. Primera aproximación al estudio de las excavaciones de 2007 ». II Jornadas de Prehistoria y Arqueología del Campo de Gibraltar. IECG. Algeciras. pp. 253-267.

GENER BASALLOTE, J. M. (1996): Informe sobre la Prospección de Urgencia en los nuevos terrenos para instalaciones de CEPSA. San Roque (Cádiz). Delegación Provincial de Cultura. Cádiz. Inédito.

GONZÁLEZ, J. (1982): Inscripciones romanas de la Provincia de Cádiz. Diputación de Cádiz. Cádiz.

- (1989): «M. Petrucidius M. f. legatus pro pr.», Athenaeum, 67. Pavia. pp. 517- 524.

GOZALBES CRAVIOTO, E. (1993): «Establecimiento de mauritanos en el Campo de Gibraltar en época de Augusto». Rev. Almoraima, 9. Algeciras. 269-276.

- (1993 bis): «Sobre el establecimiento de mauritanos en el Campo de Gibraltar en época de Augusto». Rev. Almoraima, 10. Algeciras. 44-46.

- (1997a): Economía de la Mauritania Tingitana. (Siglos I A. de C. - II D. de C.). Ceuta.

- (1997b): «La proyección económica de la Carteia romana». Rev. Almoraima, 17. Algeciras. 75-84.

HÜBNER, E. (1869): Corpus Inscriptionum Latinarum, II. Berlín. 
JIMÉNEZ-CAMINO ÁLVAREZ, R. (2002): Informe de evaluación de la afección de una obra para la construcción de un muro en el B.I.C. «Alfar Romano de El Rinconcillo». Algeciras. Inédito.

JIMENEZZ-CAMINO ÁLVAREZ, R. y BERNAL CASASOLA, D. (2007): «Redescubriendo a Traducta. Reflexiones sobre su topografía urbana y su secuencia ocupacional (ss I-VII)». Anales de Arqueología Cordobesa, 18. Córdoba. pp. 157- 200.

LAGÓSTENA BARRIOS, L. (1996): Alfarería romana de la provincia de Cádiz. Cádiz.

MARCHETTI, M. (1922): «Hispania» en DI RUGGIERO, E. Dizionario Epigrafico di Antichità Romane, III. Roma. pp. 899-917.

McWHIRR, A. (1979): «Roman tile-Kilns in Britain. Studies in manufacture, distribution and use in Western Empire». B. A. R. International Series, 68. Oxford.

MORENO, M. y VARGAS, S. (2004): «Los hornos romanos del viaducto del Pretorio» (Córdoba) en Figlinae Baeticae. Talleres alfareros y producciones cerámicas en la Bética romana (ss. II a.C - VII d. C). Facultad de Filosofía y Letras. Universidad de Cádiz. Cádiz, 12-14 noviembre 2003). (en prensa).

NY, F. Le (1988): «Les fours de tuiliers gallo-romains. Méthodologie. Étude technologique, typologique et statistique. Chronologie». Documents d'Archéologie Française, 12. París.

PÉREZ GONZÁlEZ, C. (1989): Cerámica romana de Herrera de Pisuerga (Palencia-España). La Terra Sigillata. Santiago de Chile.

PONSICH, M. (1988): Aceite de oliva y salazones de pescado. Factores socio-económicos de Bética y Tingitana. Madrid.

PRESEDO VELO, F. J., MUÑIZ COELLO, J., SANTERO SANTURINO, J. Mํa y CHAVES TRISTÁN, F. (1982): Carteia I. Excavaciones Arqueológicas en España. Madrid.

RAMBLA TORRALBO, J. A. y MAYORGA MAYORGA, J. (1997): «Hornos de época altoimperial en calle Carretería, Málaga». Figlinae Malacitanae, Málaga.

SEDEÑO FERRER, D. (1987). «Prospección arqueológica superficial realizada desde Gibraltar hasta las playas de Bolonia. Cádiz». Anuario Arqueológico de Andalucía, 1986, tomo II. Sevilla. pp. 106-109.

SERRANO RAMOS, E. (1995): «Producciones de cerámicas comunes locales de la Bética» en X. AQUILUE (ed.): Ceràmica comuna romana d'època Alto-Imperial a la Península Ibèrica Estat de la questió. Monografíes Emporitantes, VIII. pp. 227-249.

SOTOMAYOR MURO, M. (1969): «Hornos romanos de ánforas en Algeciras». X Congreso Nacional de Arqueología (Mahón, 1967). pp. 389-399.

TOMASSETTI GUERRA, J. Ma y TORREMOCHA SILVA, A. (2000): Informe preliminar de la Intervención Arqueológica de Urgencia en el Viario de la Avda. Diputación. Algeciras (Cádiz). Delegación Provincial de Cultura. Cádiz. Inédito.

SUÁREZ PADILLA, J. TOMASSETTI GUERRA, J. M., FERNÁNDEZ RODRIGUEZ, L. E. y NAVARRO LUENGO, I. (2003). «Un horno romano de época altoimperial en El SaladiIlo». Cilniana, año III, no 16. Marbella. pp. 103-112.

SUÁREZ PADILLA, J., TOMASSETTI GUERRA, J. M르, BRAVO JIMÉNEZ, S., FERNÁNDEZ RODRIGUEZ, L. E. y NAVARRO LUENGO, I. (2004): «Un horno cerámico de época altoimperial en el Saladillo (Estepona, Málaga)». Figlinae Baeticae. Talleres alfareros y producciones cerámicas en la Bética romana (ss. II a.C-VII d. C). Facultad de Filosofía y Letras. Universidad de Cádiz (Cádiz, 12-14 noviembre 2003). Cádiz. pp. 713-720.

TOMASSETTI GUERRA, J. M. y SUÁREZ PADILLA, J. (2003a): Control de Movimiento de Tierras en el Área 11-P.E.I. del P.G.O.U. de San Roque. Refinería CEPSA, San Roque (Cádiz). Informe Preliminar. Delegación Provincial de Cultura. Cádiz. Inédito.

- (2003b): Control de Movimiento de Tierras para la instalación de una tubería de hidrógeno entre la Refinería CEPSA (Guadarranque) e INTERQUISA, San Roque (Cádiz). Informe Preliminar. Delegación Provincial de Cultura. Cádiz. Inédito.

TOMASSETTI GUERRA, J. M. y BRAVO JIMÉNEZ, S. (2006): «Novedades sobre establecimientos alfareros romanos en el arco norte de la bahía de Algeciras (Cádiz)» las 
Un ejemplo de producción artesanal en el Mundo Romano: el uso de tegulae...

Jornadas de Arqueología del Campo de Gibraltar. Tarifa, 23, 24 y 25 de abril de 2004. Almoraima, 33. Algeciras. pp. $251-264$.

TOMASSETTI GUERRA. J. Ma

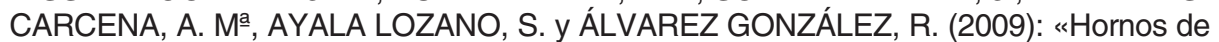
Ivlia Tradvcta (Algeciras, Cádiz): La Figlina Garavilla y su entorno paleogeográfico». Caetaria, 6-7. Algeciras. pp. 75-106.

TORREMOCHA SILVA, A., TOMASSETTI GUERRA, J. Maㅡ y JIMÉNEZ-CAMINO, R. (2000): «Excavación arqueológica de Urgencia en la Avda. Diputación. (Algeciras) y hallazgo de los restos de un horno de alfarero de época romana». Caetaria, III. Algeciras. pp. 271-272.

TREMOLEDA i TRILLA, J. (1995): «Anàlisi de l'organitzaciò dels tallers locals de ceràmica a les comarques Gironines», en X. AQUILUE (ed.): Ceràmica comuna romana d'època Alto-Imperial a la Península Ibèrica Estat de la questió. Monografíes Emporitanes, VIII. Barcelona. pp. 75-91.

VICENTE LARA, J. I. y MARFIL, P. (1991): «Nuevas perspectivas de la arqueología romana de Algeciras». Almoraima, 5. Algeciras. pp. 127-145.

ZARZALEJOS PRIETO, M. (2002): El alfar romano de Villamanta (Madrid). Universidad Autónoma. Madrid. 
22-BRAVO JIM 18/09/2012 11:19 Página 452 